\title{
Long-acting injectable paliperidone palmitate versus oral paliperidone extended release: a comparative analysis from two placebo- controlled relapse prevention studies
}

\author{
Michael Markowitz ${ }^{1 *}$, Dong-Jing Fu', Bennett Levitan², Srihari Gopal' ${ }^{2}$ Ibrahim Turkoz ${ }^{2}$ and Larry Alphs ${ }^{1}$
}

\begin{abstract}
Background: Increasing availability and use of long-acting injectable antipsychotics have generated a need to compare these formulations with their oral equivalents; however, a paucity of relevant data is available.

Methods: This post hoc comparison of the long-term efficacy, safety and tolerability of maintenance treatment with paliperidone palmitate (PP) versus oral paliperidone extended release (ER) used data from two similarly designed, randomised, double-blind (DB), placebo-controlled schizophrenia relapse prevention trials. Assessments included measures of time to relapse, symptom changes/functioning and treatment-emergent adverse events (TEAEs). Time to relapse between treatment groups was evaluated using a Cox proportional hazards model. Between-group differences for continuous variables for change scores during the DB phase were assessed using analysis of covariance models. Categorical variables were evaluated using Chi-square and Fisher's exact tests. No adjustment was made for multiplicity.

Results: Approximately $45 \%$ of enrolled subjects in both trials were stabilised and randomised to the DB relapse prevention phase. Risk of relapse was higher in subjects treated with paliperidone ER than in those treated with PP [paliperidone ER/PP hazard ratio (HR), 2.52; 95\% confidence interval (Cl), 1.46-4.35; $p<0.001$ ]. Similarly, risk of relapse after withdrawal of paliperidone ER treatment (placebo group of the paliperidone ER study) was higher than after withdrawal of PP (paliperidone ER placebo/PP placebo HR, 2.25; 95\% Cl, 1.59-3.18; $p<0.001$ ). Stabilised schizophrenic subjects treated with PP maintained functioning demonstrated by the same proportions of subjects with mild to no difficulties in functioning at DB baseline and end point [Personal and Social Performance (PSP) scale total score $>70$, both approximately $58.5 \% ; p=1.000$ ] compared with a 10.9\% decrease for paliperidone ER ( $58.5 \%$ vs $47.6 \%$, respectively; $p=0.048$ ). The least squares mean change for Positive and Negative Syndrome Scale (PANSS) total score at DB end point in these previously stabilised subjects was 3.5 points in favour of PP (6.0 vs 2.5; $p=0.025)$. The rates of TEAEs and AEs of interest appeared similar.

Conclusions: This analysis supports maintenance of effect with the injectable compared with the oral formulation of paliperidone in patients with schizophrenia. The safety profile of PP was similar to that of paliperidone ER. Future studies are needed to confirm these findings.
\end{abstract}

Keywords: Post hoc analysis, Paliperidone extended release, Long-acting injectable, Paliperidone palmitate

\footnotetext{
* Correspondence: mmarkowi@its.jnj.com

'Janssen Scientific Affairs, LLC, 1125 Trenton-Harbourton Road, Titusville, NJ 08560, USA

Full list of author information is available at the end of the article
} 


\section{Background}

Long-term maintenance therapy in schizophrenia is an important clinical and public health concern requiring careful balance between benefits and risks. This problem is important on both an individual patient level and a societal public health level, as the medical and societal costs of relapsing patients are high [1].

A major obstacle to the effective treatment of patients with schizophrenia is non-adherence with the medication regimen. It is estimated that 1 -year rates of treatment discontinuation or interruption range from $40 \%$ to $75 \%$ [2,3]. Individual patients may discontinue or interrupt treatment as the result of a variety of factors, such as lack of illness insight, forgetfulness, lack of social support, tolerability issues, conscious choice and refractory or non-responsive symptoms [4,5]. Long-acting injectable (LAI) medications can help overcome problems with non-adherence by removing the need for daily dosing and by simplifying treatment; additionally, the healthcare provider can know with certainty whether a patient has received an injection and can make an appropriate intervention if the patient has not [6]. Several studies have shown that switching from an oral to an LAI antipsychotic is both safe and effective [7-10]. Unfortunately, the few head-to-head studies that have compared oral and injectable antipsychotics in terms of safety and efficacy have design limitations such as a short duration of treatment or a small sample size $[11,12]$. Long-term head-to-head comparisons against alternative oral treatment approaches have not been done [7-10]. To provide some basis for comparing the formulations, we conducted a post hoc indirect comparative efficacy analysis aimed at comparing long-term efficacy, including relapse prevention, and safety/tolerability of once-monthly paliperidone palmitate (PP) against oral paliperidone extended release (ER) using indirect comparative techniques and subject-level data from two similarly designed maintenance treatment studies $[13,14]$.

\section{Methods}

\section{Indirect comparison}

In the absence of head-to-head trials comparing the long-term efficacy and safety of ER and PP, patient-level data on maintenance treatment from two similarly designed relapse studies were used $[13,14]$ to conduct an indirect comparison for this post hoc analysis. Metaanalysis of relapse studies of other antipsychotics in the literature was not pursued because of widely varying study criteria and differences in the definition of relapse. It is known that differences in the definition of relapse significantly affect identified relapse rates [15]. The standard approach for an indirect comparison accounts for some difference between trials by placebo adjustment.
However, because the LAI stays in the bloodstream for months compared with days for the oral ER formulation [16], the group that is withdrawn from PP and given a placebo still has active treatment in their bloodstream for several months [terminal half-life $\left(\mathrm{t}_{1 / 2}\right), 25$ to 49 days, depending on the dose] [17], while the group given an oral placebo has no substantial active treatment after 4 to 5 days $\left(t_{1 / 2}=23 \mathrm{~h}\right)$. Placebo-corrected comparisons therefore are not meaningful in this randomised withdrawal setting because of the dramatically different pharmacokinetics of the formulations. For this work, we have compared active with active and placebo with placebo directly. As discussed later, this approach is supported by similarity of design and baseline patient properties in the trials selected.

\section{Identification of source data}

Two similarly designed randomised, double-blind (DB), placebo-controlled schizophrenia relapse prevention studies of paliperidone ER (NCT00086320) [13] and PP (NCT00111189) [14] were included (Figure 1). These trials had comparable study designs (i.e. run-in/transition, stabilisation, DB and optional open-label extension phases), comparable stabilisation and relapse criteria and similar inclusion and exclusion criteria. The paliperidone ER study had an 8-week run-in phase followed by a 6week open-label stabilisation phase [13], and the PP trial had a 9-week run-in phase followed by a 24-week openlabel stabilisation phase [14]. In both trials, subjects were first stabilised with paliperidone ER or PP [defined by Positive and Negative Syndrome Scale (PANSS) total scores $\leq 75$ and select PANSS item scores $\leq 4$ ] and were then randomly assigned to active treatment or placebo. The DB phase was variable in the two trials: subjects remained in the DB phase until they experienced a relapse, until they withdrew from the study or until the study was completed. Therefore, the length of time in the DB period differed for each patient. Based on significant improvements over placebo, both studies were terminated early. Analysis was performed on the DB relapse prevention phase of the two studies, using the DB intent-to-treat analysis set, consisting of all randomly assigned subjects who received at least one dose of DB medication. Both original studies were conducted in accordance with the ethical principles that have their origin in the Declaration of Helsinki and that are consistent with Good Clinical Practices and applicable regulatory requirements. The original study protocols were reviewed and approved by an independent ethics committee or an institutional review board at each study site, and all subjects provided written informed consent before entering the studies.

The two studies had similar but slightly different stabilisation criteria. To match their randomisation in this 


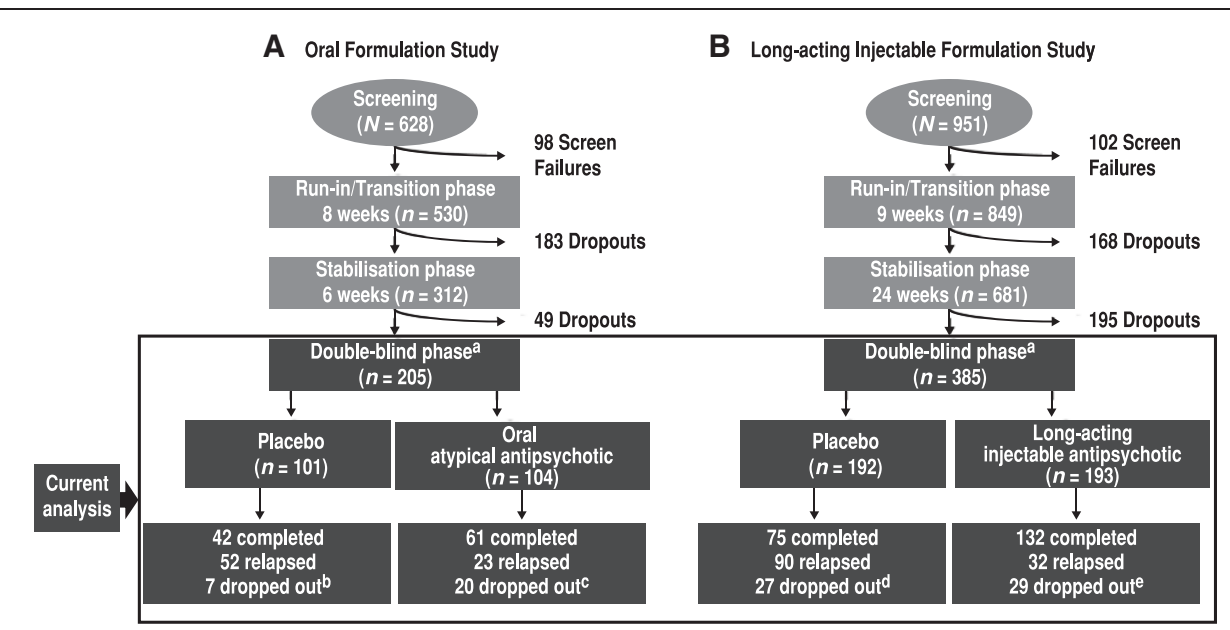

Figure 1 Designs of the two studies used in the analysis. A Paliperidone extended release (ER) vs placebo study. B Paliperidone palmitate (PP) vs placebo study. ${ }^{a}$ Subjects remained in the double-blind phase until they experienced a relapse, until they withdrew from the study or until the study was completed. The double-blind phase was followed by an open-label extension in each study. Based on significant efficacy, both studies were terminated early. At the time the studies were stopped, a total of 91 subjects in the paliperidone ER study and a total of 76 subjects in the PP study were in the stabilisation phase and were considered as completing the entire study per protocol. Subjects $(n=25)$ in the PP study who did not meet the more stringent stabilisation criteria employed in the paliperidone ER study were excluded to standardise the study

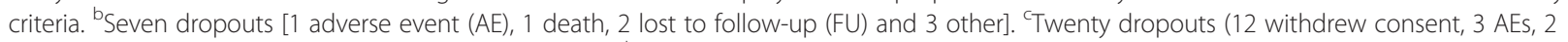
lost to FU, 1 study drug protocol violation and 2 other). ${ }^{\mathrm{d}}$ Twenty-seven dropouts (15 withdrew consent, 2 AEs and 10 other). ${ }^{\mathrm{e}} \mathrm{Tw}$ wenty-nine dropouts (12 withdrew consent, 3 AEs and 14 other).

analysis, subjects $(n=25)$ were excluded from the PP study because they did not meet the more stringent measures of PANSS $\leq 70$ and Clinical Global Impressions-Severity (CGI-S) $\leq 4$ (not ill to moderately ill), which were employed as stabilisation criteria in the oral formulation study.

\section{Definition of outcomes}

This post hoc analysis was performed on efficacy and safety measures during the $\mathrm{DB}$ phase of the two studies.

Relapse during the clinical trials was defined as the first occurrence of one or more of the following:

- Psychiatric hospitalisation (involuntary or voluntary admission)

- Increase of $25 \%$ in PANSS total score for two consecutive assessments for subjects who scored $>40$ at randomisation, or a 10-point increase for subjects who scored $\leq 40$ at randomisation

- Deliberate self-injury or aggressive behaviour, or suicidal or homicidal ideation and aggressive behaviour that was clinically significant

- Increase for two consecutive assessments in prespecified individual PANSS item scores (P1, P2, P3, P6, P7 and G8) to $\geq 5$ for subjects whose score was $\leq 3$ at randomisation, or to $\geq 6$ for subjects whose score was 4 at randomisation

In the paliperidone ER study only [13], relapse was also defined as an increase in CGI-S score to $\geq 4$ for subjects who scored $\leq 3$ at randomisation, or to $\geq 5$ for subjects whose score was 4 at randomisation for two consecutive assessments. Subjects who met the CGI-S criterion also met the other relapse criteria; therefore, no adjustment in relapse definition was necessary for this analysis. Definitions of maintenance phase outcomes, other than relapse, are shown in Table 1.

\section{Statistical methods}

Demographic and baseline characteristics were summarised using descriptive statistics for the DB phase of each study. The baseline demographic and disease characteristics of both studies were also compared to identify potential confounders. Time to relapse between treatment groups was evaluated using Cox proportional hazards analysis and the log-rank test. The cumulative distribution function of the time to relapse was estimated by the Kaplan-Meier method. Between-group differences for continuous variables for change scores during the DB phase were assessed using analysis of co-variance models with baseline values as the continuous co-variate and using the last observation carried forward method for imputation of missing values. Categorical variables were evaluated using odds ratios, Chi-square tests and Fisher's exact test. Shifts from baseline to end point in Personal and Social Performance (PSP) categories were examined using McNemar's test. Treatment-emergent adverse event (TEAE) rates were also compared. No adjustment was made for multiplicity. 
Table 1 Efficacy and safety outcomes

\begin{tabular}{ll}
\hline Outcome & Measures and definitions $^{\mathbf{a}}$ \\
\hline Efficacy & \\
CGI-S & Change in CGI-S score \\
PANSS & Change in total PANSS scores \\
Relapse & Time to first relapse and percentage relapse \\
PSP & Change in PSP total score \\
& Frequency of PSP category change (categories 0 to 70, 71 \\
& to 100) \\
Safety & \\
AEs & Overall incidence, $n$ (\%) \\
& Serious AEs, $n$ (\%) \\
& AEs leading to discontinuation, $n$ (\%) \\
Weight & AEs of interest (EPS-related and prolactin-related AEs), $n$ (\%) \\
gain &
\end{tabular}

${ }^{\mathrm{a}}$ From double-blind baseline to double-blind end point. $A E$ adverse event, CGI-S Clinical Global Impressions-Severity, EPS extrapyramidal symptoms, PANSS Positive and Negative Syndrome Scale, PSP Personal and Social Performance.

\section{Results}

Baseline demographic and clinical characteristics of the two study populations appeared comparable except for race because the studies were conducted in different countries (Table 2). A total of 385 subjects were included in the PP study (193 PP, 192 placebo), and 205 in the paliperidone ER study (104 paliperidone ER, 101 placebo). Table 3 summarises the extent of exposure of the two studies. As described in the original studies $[13,14]$, both trials were stopped after interim analyses as a result of significant benefit of PP and ER over placebo. These early terminations resulted in median duration of exposure in the DB phase of 170 days (range 1 to 407 days) for the PP study versus 45 days (range 3 to 330 days) for the paliperidone ER study.

\section{Efficacy}

Patients were required to be stable (defined as PANSS total scores $\leq 75$ and select PANSS item scores $\leq 4$ ) to enter the DB period. Therefore, efficacy was measured by the degree to which this stability was lost, which was a result of relapse and corresponding efficacy and functionality findings.

The two previously conducted clinical trials provide the basis for this post hoc comparison. Both showed active treatment to be superior to placebo in schizophrenia relapse studies (Figure 2) [13,14].

In this indirect comparative analysis of active treatment groups, the risk of relapse was higher in subjects treated with paliperidone ER than in subjects treated with PP (paliperidone ER/PP HR 2.52; 95\% CI 1.46 to 4.35; $p<0.001$ ) (Table 4). Similarly, the risk of relapse

Table 2 Double-blind baseline demographics and clinical characteristics

\begin{tabular}{|c|c|c|c|c|}
\hline & \multicolumn{2}{|c|}{ Study active arms } & \multicolumn{2}{|c|}{ Study placebo arms } \\
\hline & $\begin{array}{c}\mathrm{PP} \\
(n=193)\end{array}$ & $\begin{array}{l}\text { Paliperidone ER } \\
\quad(n=104)\end{array}$ & $\begin{array}{l}\text { PP placebo } \\
(n=192)\end{array}$ & $\begin{array}{l}\text { Paliperidone ER placebo } \\
\qquad(n=101)\end{array}$ \\
\hline Age in years, mean (SD) & $38.6(11.4)$ & $39.0(10.7)$ & $39.2(10.8)$ & $37.5(10.4)$ \\
\hline \multicolumn{5}{|l|}{ Sex, $n(\%)$} \\
\hline Male & $104(53.9)$ & $58(55.8)$ & $104(54.2)$ & $63(62.4)$ \\
\hline Female & $89(46.1)$ & $46(44.2)$ & $88(45.8)$ & $38(37.6)$ \\
\hline \multicolumn{5}{|l|}{ Race, $n(\%)$} \\
\hline Caucasian & $124(64.3)$ & $62(59.6)$ & $126(65.6)$ & $61(60.4)$ \\
\hline Black & $36(18.7)$ & $8(7.7)$ & $35(18.2)$ & $9(8.9)$ \\
\hline Asian & $30(15.5)$ & $3(2.9)$ & $27(14.1)$ & 0 \\
\hline Other & $3(1.6)$ & $31(29.8)^{a}$ & $4(2.1)$ & $31(30.7)^{a}$ \\
\hline Age at diagnosis in years, mean (SD) & $26.3(9.2)$ & $27.1(9.2)$ & $28.2(8.9)$ & $25.8(9.4)$ \\
\hline Baseline PANSS total score, mean (SD) & $50.8(11.0)$ & $51.0(11.4)$ & $51.9(11.1)$ & $53.4(10.6)$ \\
\hline \multicolumn{5}{|l|}{ Baseline CGI-S score, $n(\%)$} \\
\hline Not ill & $11(5.7)$ & $6(5.8)$ & $11(5.7)$ & $5(5.0)$ \\
\hline Very mildly ill & 69 (35.8) & $38(36.5)$ & $77(40.1)$ & $33(32.7)$ \\
\hline Mildly ill & $92(47.7)$ & $49(47.1)$ & $81(42.2)$ & $54(53.5)$ \\
\hline Moderately ill & $21(10.9)$ & $11(10.6)$ & $23(12.0)$ & $9(8.9)$ \\
\hline Previous hospitalisations for psychosis, mean (SD) & $2.6(1.2)^{\mathrm{a}}$ & $2.9(1.2)^{\mathrm{b}}$ & $2.7(1.2)^{\mathrm{a}}$ & $2.9(1.2)^{c}$ \\
\hline
\end{tabular}

${ }^{a} n=173 ;{ }^{b} n=78 ;{ }^{c} n=74$. CGI-S Clinical Global Impressions-Severity, ER extended release, PANSS Positive and Negative Syndrome Scale, $P P$ paliperidone palmitate, $S D$ standard deviation. 
Table 3 Extent of exposure

\begin{tabular}{lcc}
\hline & PP & Paliperidone ER \\
\hline $\begin{array}{l}\text { Median duration, } \\
\text { days (range) }\end{array}$ & 170 (1 to 407) & 45 (3 to 330) \\
$\begin{array}{l}\text { Mean (SD) dose } \\
\begin{array}{l}\text { Mode monthly/daily dose } \\
\text { (percentage of subjects) }\end{array}\end{array}$ & $128.0(38.7) \mathrm{mg} / \mathrm{month}$ & 10.8 (3.3) mg/day \\
\hline ER extended release, $P$ P paliperidone palmitate. & $9 \mathrm{mg} /$ day (38)
\end{tabular}

$E R$ extended release, $P P$ paliperidone palmitate. after withdrawal of paliperidone ER treatment (placebo group of the paliperidone ER study) was higher than after withdrawal of PP (paliperidone ER placebo/PP placebo HR 2.25; 95\% CI 1.59 to $3.18 ; p<0.0001)$. The most common reason for relapse was an increase in total PANSS of $\geq 25 \%$ (Figure 3 ).

Efficacy results for PANSS, PSP and CGI-S, comparing $\mathrm{PP}$ and paliperidone ER treatment, are shown in Table 5. During the DB phase, both PP and paliperidone ER treatment groups showed slight worsening in efficacy outcomes from baseline (i.e. increase in PANSS total

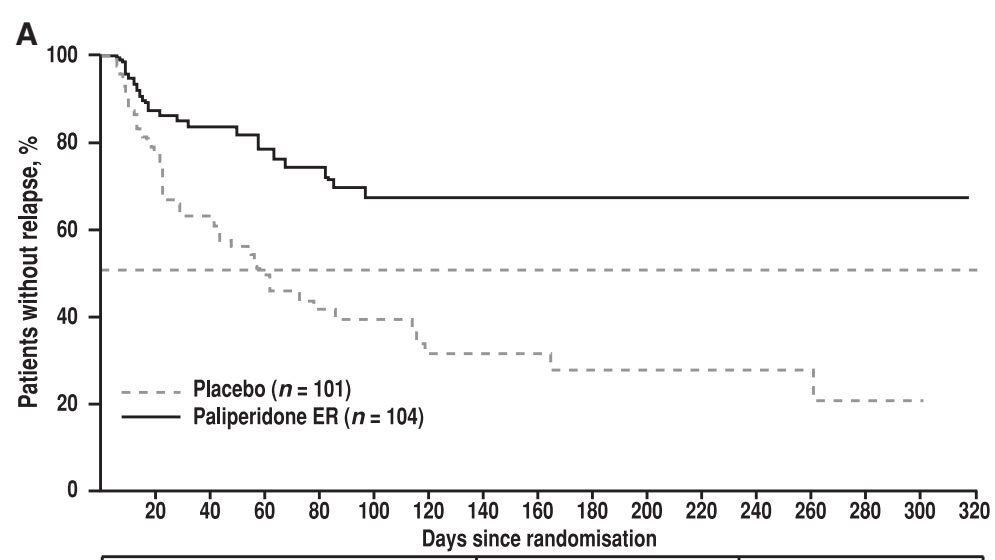

\begin{tabular}{|c|c|c|}
\hline & Paliperidone ER & Placebo \\
\hline$N$ & 104 & 101 \\
\hline Patients with events, $\boldsymbol{n}(\%)$ & $23(22)$ & $52(51)$ \\
\hline K-M median days (95\% Cl) & NA & $58(44$ to 14$)$ \\
\hline K-M $25^{\text {th }}$ percentile, days $(95 \% \mathrm{Cl})$ & 68 (50 to NA) & $23(15$ to 29$)$ \\
\hline Hazard ratio $(95 \% \mathrm{Cl})$ & \multicolumn{2}{|c|}{$2.83(1.73$ to 4.63$)$} \\
\hline Log-rank test & \multicolumn{2}{|c|}{$p<0.001$} \\
\hline
\end{tabular}

NA: Computations cannot be made because not enough events were observed.

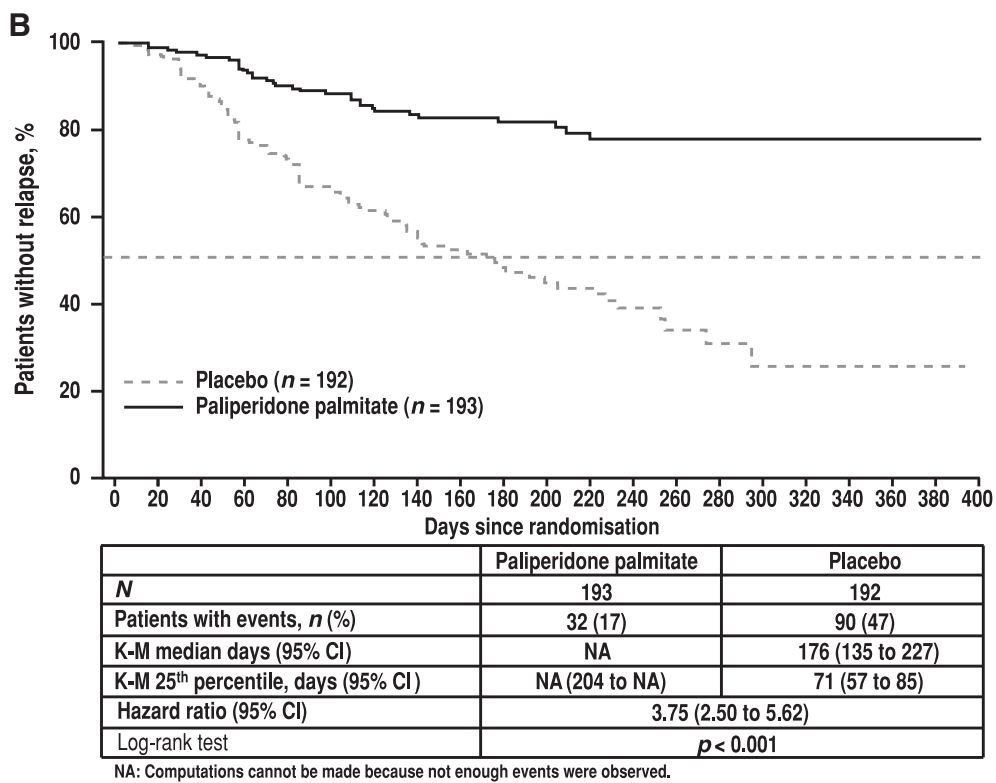

Figure 2 Kaplan-Meier curves for relapse in treatment and placebo groups. (A) Paliperidone extended release (ER). (B) Paliperidone palmitate. Cl confidence interval, ER extended release, K-M Kaplan-Meier. 
Table 4 Indirect comparison of hazard ratios for risk of relapse

\begin{tabular}{|c|c|c|c|c|}
\hline & $\begin{array}{c}\text { PP } \\
(n=193)\end{array}$ & $\begin{array}{l}\text { Paliperidone ER } \\
\quad(n=104)\end{array}$ & $\begin{array}{l}\text { PP placebo } \\
(n=192)\end{array}$ & $\begin{array}{l}\text { Paliperidone ER placebo } \\
\qquad(n=101)\end{array}$ \\
\hline Subjects with events, $n(\%)$ & $32(17)$ & $23(22)$ & $90(47)$ & $52(51)$ \\
\hline Hazard ratio $(95 \% \mathrm{Cl})^{\mathrm{a}}$ & \multicolumn{2}{|c|}{2.52 (1.46 to 4.35$)$} & \multicolumn{2}{|c|}{2.25 (1.59 to 3.18$)$} \\
\hline
\end{tabular}

${ }^{\mathrm{a}}$ Hazard ratios reflect paliperidone ER/PP for active treatments and placebo treatments, respectively. $\mathrm{Cl}$ confidence interval, $E R$ extended release, $P P$ paliperidone palmitate.

scores and CGI-S scores) and in functioning outcomes (i.e. decrease in PSP total scores). The least squares (LS) mean change for the PANSS total score at DB end point (6.0 for the paliperidone ER study and 2.5 for the PP study) was 3.5 points in favour of PP and was statistically significant $(p=0.025)$, indicating that on average, subjects treated with PP demonstrated better maintenance of their DB baseline status than those treated with the oral formulation. This difference was evident, even though the period of observation for the LAI treatment group was longer than for the paliperidone ER treatment group (median 170 days for the PP study vs 45 days for paliperidone ER). No significant between-group differences were noted in the change in mean PSP or CGI-S scores from baseline. However, less decline in functional status was observed, as measured by PSP in subjects treated with PP (Figure 4); those treated with PP demonstrate virtually no difference between DB baseline and end point in the proportion of subjects with mild to no difficulties in functioning (PSP score $>70 ; 58.5 \%$ vs $58.5 \%$, respectively; $p=1.000$ ), compared with a $10.9 \%$ decrease for paliperidone ER (58.3\% vs $47.6 \%$, respectively; $p=0.048)$.

\section{Safety}

What appeared to be similar incidences of adverse events (AEs) of interest [extrapyramidal symptoms (EPS)-related AEs, prolactin-related AEs and weight increase] were observed (PP and paliperidone ER, respectively): low incidence of AEs leading to discontinuation (1.6\% vs $2.9 \%) ; \geq 1$ serious treatment-emergent $\mathrm{AE}$ (4.2\% vs $7.7 \%$ ); EPS-related AEs (5.7\% vs 6.7\%); prolactin-related AEs (2.1\% vs $2.9 \%$ ) and weight increase $\geq 7 \%$ ( $23.4 \%$ vs $19.6 \%$ ) (Table 6 ).

\section{Discussion}

Results from the two previously published studies show that differences between treatment groups in time to relapse significantly favoured paliperidone ER and PP, compared with placebo. The indirect comparison between studies described here suggests that the risk of relapse was significantly higher in subjects treated with paliperidone ER as compared with subjects treated with PP (paliperidone ER/PP HR 2.52; 95\% CI 1.46 to $4.35 ; p<$ 0.001). Similarly, the ER placebo group had a higher risk of relapse than the PP placebo group. These placebo results are likely due to the shorter half-life of paliperidone

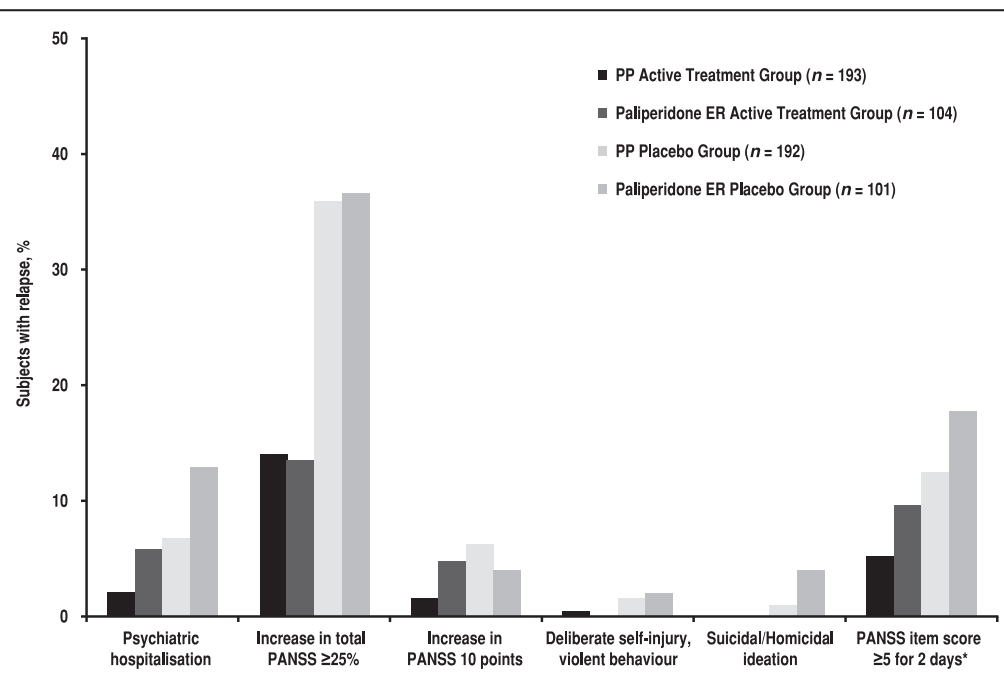

Figure 3 Frequency of different types of relapse during double-blind phase (entire exposure period). Categories are not mutually exclusive; some subjects met $>1$ relapse criterion. Asterisk indicates increase for two consecutive assessments in pre-specified individual PANSS item scores to $\geq 5$ for subjects whose score was $\leq 3$ at randomisation. ER extended release, PANSS Positive and Negative Syndrome Scale, PP paliperidone palmitate. 
Table 5 Efficacy outcomes from the double-blind period

\begin{tabular}{lccc}
\hline Efficacy measure & PP & $\begin{array}{c}p \text { Paliperidone } \\
\text { ER }\end{array}$ & $\begin{array}{c}p \text { Value } \\
\text { (paliperidone ER vs PP) }\end{array}$ \\
\hline PANSS total score & $n=191$ & $n=104$ \\
Double-blind baseline mean (SD) & $50.8(10.9)$ & $51.0(11.4)$ \\
LS mean (SE) change from baseline to end point & $2.5(0.9)$ & $6.0(1.3)$ & $n=103$ \\
PSP score & $n=188$ & $70.8(10.9)$ \\
Double-blind baseline mean (SD) & $72.7(10.5)$ & $-3.3(1.1)$ & 0.025 \\
LS mean (SE) change from baseline to end point & $-1.4(0.8)$ & $n=104$ \\
CGI-S score & $n=191$ & $2.6(0.8)$ \\
Double-blind baseline mean (SD) & $2.6(0.8)$ & $0.3(0.1)$ & 0.156 \\
LS mean (SE) change from baseline to end point & $0.2(0.1)$ & 0.095 \\
\hline
\end{tabular}

CGI-S Clinical Global Impressions-Severity, ER extended release, LS least squares, PANSS Positive and Negative Syndrome Scale, PP paliperidone palmitate, PSP Personal and Social Performance, SD standard deviation, SE standard error.

ER compared with that of paliperidone LAI. As a consequence, the LAI placebo group continued to receive benefit from residual plasma concentrations of paliperidone for many weeks after the final injection of PP.

The LS mean change for the PANSS total score at end point was 3.5 points lower for PP $(p=0.025)$, despite a nearly fourfold longer period of observation for the PP group. Results of the PSP evaluation suggest that improved symptomatic outcome with PP translates to better maintenance of functioning.

Both PP and paliperidone ER were well tolerated. What appeared to be similar rates of AEs of interest were observed for the two formulations, and no additional safety concerns were associated with LAI versus oral paliperidone. Indeed, the numerical incidence rates favoured PP for all AEs other than weight gain despite the fourfold longer period of exposure for the PP group. The greater weight gain in the PP group may have been related to the longer period of exposure and increased time to gain weight.

This study has several limitations: (1) It was not possible to compare results from these studies by examining differences from placebo treatment because of the large differences in pharmacokinetic properties. The LAI formulation used in the stabilisation phase remained in the plasma for several months (median apparent $\mathrm{t}^{1 / 2} 25$ to 49 days, depending on dose), whereas the oral formulation was cleared in days (median $\mathrm{t}^{1 / 2} 23 \mathrm{~h}$ ). Thus, for several months after the start of the DB phase, the placebo group formerly on the LAI still was effectively on treatment and those in the placebo arm of the oral formulation study were not. (2) These studies used slightly

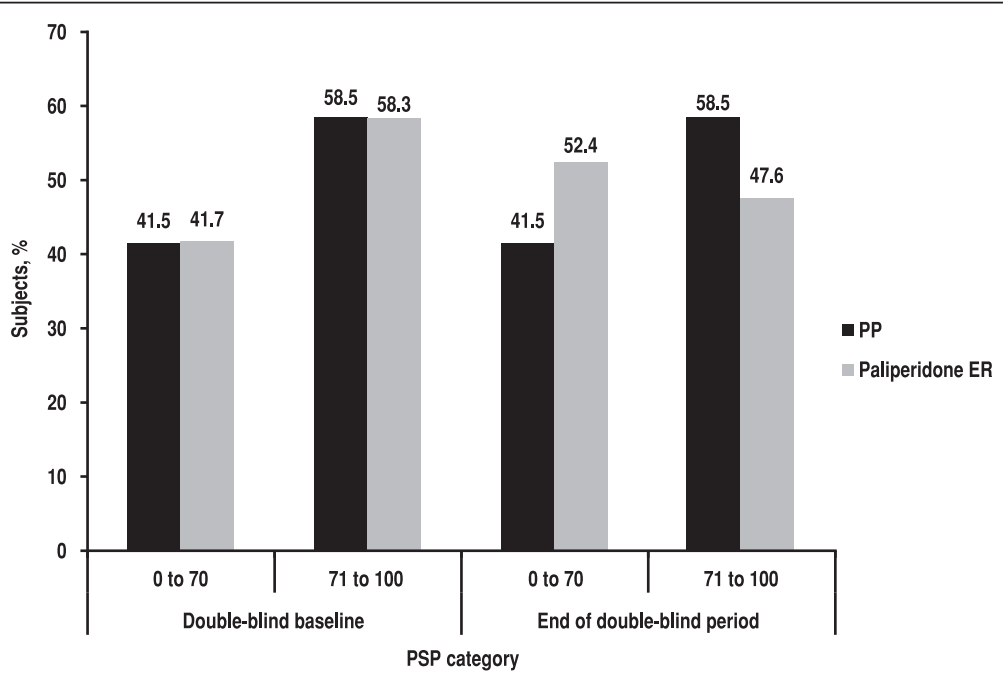

Figure 4 Frequency of Personal and Social Performance (PSP) categories of score during double-blind phase. $E R$ extended release, $P P$ paliperidone palmitate. 
Table 6 Adverse events summary from the double-blind period

\begin{tabular}{lcccc}
\hline & $\begin{array}{c}\text { PP } \\
(\boldsymbol{n}=\mathbf{1 9 3})\end{array}$ & $\begin{array}{c}\text { Paliperidone } \\
\text { ER } \\
(\boldsymbol{n}=\mathbf{1 0 4})\end{array}$ & $\begin{array}{c}\text { PP } \\
\text { placebo } \\
(\boldsymbol{n}=\mathbf{1 9 2})\end{array}$ & $\begin{array}{c}\text { Paliperidone } \\
\text { ER placebo } \\
(\boldsymbol{n}=\mathbf{1 0 1})\end{array}$ \\
\hline All TEAEs, $n(\%)$ & $83(43.0)$ & $36(34.6)$ & $84(43.8)$ & $41(40.6)$ \\
$\geq 1$ serious TEAE & $8(4.2)$ & $8(7.7)$ & $23(12.0)$ & $16(15.8)$ \\
$\begin{array}{l}\text { TEAE leading to } \\
\text { discontinuation }\end{array}$ & $3(1.6)$ & $3(2.9)$ & $1(0.5)$ & $1(1.0)$ \\
$\begin{array}{l}\text { EPS-related AEs } \\
\text { Prolactin-related }\end{array}$ & $4(2.1)$ & $3(2.9)$ & $3(1.6)$ & 0 \\
AEs & & $7(6.7)$ & $3(1.6)$ & $3(3.0)$ \\
$\begin{array}{l}\text { Weight } \\
\text { increase } \geq 7 \%\end{array}$ & $44(23.4)$ & $19(19.6)$ & $24(13.0)$ & $11(11.7)$ \\
\hline
\end{tabular}

$A E$ adverse event, $E P S$ extrapyramidal symptoms, $E R$ extended release, $P P$ paliperidone palmitate, TEAE treatment-emergent AE.

different definitions of relapse; however, the most common reason for relapse in all groups was an increase in total PANSS of $\geq 25 \%$. Subjects who met the additional criterion in the paliperidone ER study also met the other relapse criteria; therefore, no adjustment to the relapse definition was necessary. (3) The median duration of exposure in the DB phase was 171 days for the PP study versus 45 days for the paliperidone ER study. It is not possible to correct for differences in exposure, as exposure differences were due to trial design, different pharmacokinetic parameters of paliperidone ER and paliperidone palmitate and efficacy differences (both studies stopped early after interim analyses, and patients in the ER group relapsed sooner). Also, the impact on AEs is complex because the rate of occurrence of adverse events is not constant over time (AEs tend to occur more frequently early after exposure rather than late in the trial). (4) The combined run-in/transition/ stabilisation phases were longer for the PP study than for the paliperidone ER study (33 weeks vs 14 weeks, respectively); therefore, subjects in the PP study had maintained stabilisation for a longer period. This may have resulted in a greater level of stability in the PP group at the time of randomisation. Additionally, the paliperidone ER study required subjects to experience an acute episode at study entry, whereas the PP study did not. These differences in inclusion criteria may have influenced the study results. (5) The dose range used in the paliperidone ER study was 3 to $15 \mathrm{mg} /$ day, which is higher than the approved range (3 to $12 \mathrm{mg} /$ day). In contrast, the allowable dose range in the PP study was 39 to $156 \mathrm{mg}$ ( 25 to $100 \mathrm{mg}$ eq), which roughly equates to 2 to $8 \mathrm{mg} /$ day of paliperidone $\mathrm{ER}$ and is narrower than the approved range of 39 to $234 \mathrm{mg}$ ( 25 to $150 \mathrm{mg}$ eq). This difference in dose could potentially bias the results of the study in favour of paliperidone ER. (6)
Higher proportions of African American subjects (19\% vs $8 \%$ ) and Asian subjects (16\% vs $3 \%$ ) and a lower proportion of other subjects ( $2 \%$ vs $30 \%)$ were treated with PP compared with subjects treated with paliperidone ER. It is unknown whether these racial differences may have affected the study results.

\section{Conclusions}

This analysis supports maintenance of effect with the injectable compared with the oral formulation of paliperidone in patients with schizophrenia. This finding may be due to the adherence advantage of LAI over the oral agent. The prolonged release characteristics of PP may provide a longer protective advantage over paliperidone ER in subjects at risk for abrupt discontinuation. Despite the long-acting nature of $\mathrm{PP}$, its $\mathrm{AE}$ profile appeared similar to that of paliperidone ER. Future studies are needed to confirm these findings.

\section{Abbreviations}

AE: Adverse event; CGI-S: Clinical Global Impressions-Severity; DB: Doubleblind; Cl: Confidence interval; EPS: Extrapyramidal symptoms; ER: Extended release; HR: Hazard ratio; LAl: Long-acting injectable; LS, Least squares; PANSS: Positive and Negative Syndrome Scale; PP: Paliperidone palmitate; PSP: Personal and Social Performance; $t_{1 / 2}$ : Terminal half-life; TEAE: Treatmentemergent adverse event.

\section{Competing interests}

This work was funded by Janssen Scientific Affairs, LLC. MM, DJF and LA are employees of Janssen Scientific Affairs, LLC, and are Johnson \& Johnson stockholders. BL, SG and IT are employees of Janssen Research \& Development, LLC, and are Johnson \& Johnson stockholders.

\section{Authors' contributions}

MM contributed to study conceptualisation and management, study design data analysis and writing. DJF, BL and LA contributed to study design, data analysis and writing. SG contributed to study design, data analysis, data collection and writing. IT contributed to study design, data collection, writing and statistical analyses. All authors critically reviewed and revised the manuscript and approved the final manuscript.

\section{Acknowledgements}

The authors acknowledge Matthew Grzywacz, PhD, Sheena Hunt, PhD, and ApotheCom (supported by Janssen Scientific Affairs, LLC) for providing writing and editorial assistance.

\section{Author details}

'Janssen Scientific Affairs, LLC, 1125 Trenton-Harbourton Road, Titusville, NJ 08560, USA. ${ }^{2}$ Janssen Research \& Development, LLC, 1125 TrentonHarbourton Road, Titusville, NJ 08560, USA.

Received: 30 April 2013 Accepted: 30 June 2013

Published: 11 July 2013

\section{References}

1. Csernansky JG, Schuchart EK: Relapse and rehospitalisation rates in patients with schizophrenia: effects of second generation antipsychotics. CNS Drugs 2002, 16:473-484.

2. Bhanji $\mathrm{NH}$, Chouinard $\mathrm{G}$, Margolese $\mathrm{H}$ : A review of compliance, depot intramuscular antipsychotics and the new long-acting injectable atypical antipsychotic risperidone in schizophrenia. Eur Neuropsychopharmacol 2004, 14:87-92.

3. Masand PS, Roca M, Turner MS, Kane JM: Partial adherence to antipsychotic medication impacts the course of illness in patients with schizophrenia: a review. Prim Care Companion J Clin Psychiatry 2009, 11:147-154 
4. Tiihonen J, Haukka J, Taylor M, Haddad PM, Patel MX, Korhonen P: A nationwide cohort study of oral and depot antipsychotics after first hospitalization for schizophrenia. Am J Psychiatry 2011, 168:603-609.

5. Lacro JP, Dunn LB, Dolder CR, Leckband SG, Jeste DV: Prevalence of and risk factors for medication nonadherence in patients with schizophrenia: a comprehensive review of recent literature. J Clin Psychiatry 2002, 63:892-909.

6. Weiden PJ, Schooler NR, Weedon JC, Elmouchtari A, Sunakawa A, Goldfinger SM: A randomized controlled trial of long-acting injectable risperidone vs continuation on oral atypical antipsychotics for first-episode schizophrenia patients: initial adherence outcome. J Clin Psychiatry 2009, 70:1397-1406.

7. Lindenmayer JP, Eerdekens E, Berry SA, Eerdekens M: Safety and efficacy of long-acting risperidone in schizophrenia: a 12-week, multicenter, open-label study in stable patients switched from typical and atypical oral antipsychotics. J Clin Psychiatry 2004, 65:1084-1089.

8. Hawley C, Turner M, Latif MA, Curtis V, Saleem PT, Wilton K: Switching stable patients with schizophrenia from depot and oral antipsychotics to long-acting injectable risperidone: reasons for switching and safety. Hum Psychopharmacol 2010, 25:37-46.

9. Kim SW, Shin IS, Kim JM, Lee SH, Lee YH, Yang SJ, Yoon JS: Effects of switching to long-acting injectable risperidone from oral atypical antipsychotics on cognitive function in patients with schizophrenia. Hum Psychopharmacol 2009, 24:565-573.

10. Lloyd K, Latif MA, Simpson S, Shrestha KL: Switching stable patients with schizophrenia from depot and oral antipsychotics to long-acting injectable risperidone: efficacy, quality of life and functional outcome. Hum Psychopharmacol 2010, 25:243-252.

11. Chue P, Eerdekens M, Augustyns I, Lachaux B, Molcan P, Eriksson L, Pretorius H, David AS: Comparative efficacy and safety of long-acting risperidone and risperidone oral tablets. Eur Neuropsychopharmacol 2005, 15:111-117.

12. Bai YM, Chen TT, Wu B, Hung CH, Lin WK, Hu TM, Lin CY, Chou P: A comparative efficacy and safety study of long-acting risperidone injection and risperidone oral tablets among hospitalized patients: 12-week randomized, single-blind study. Pharmacopsychiatry 2006, 39:135-141.

13. Kramer M, Simpson G, Maciulis V, Kushner S, Vijapurkar U, Lim P, Eerdekens M: Paliperidone extended-release tablets for prevention of symptom recurrence in patients with schizophrenia: a randomized, double-blind, placebo-controlled study. J Clin Psychopharmacol 2007, 27:6-14.

14. Hough D, Gopal S, Vijapurkar U, Lim P, Morozova M, Eerdekens M: Paliperidone palmitate maintenance treatment in delaying the time-to -relapse in patients with schizophrenia: a randomized, double-blind, placebo-controlled study. Schizophr Res 2010, 116:107-117.

15. Knapp M, Locklear J, Jarbrink K: Impact of psychotic relapse definitions in assessing drug efficacy and costs: comparison of quetiapine XR, olanzapine and paliperidone ER. Curr Med Res Opin 2009, 25:1593-1603.

16. Samtani MN, Vermeulen A, Stuyckens K: Population pharmacokinetics of intramuscular paliperidone palmitate in patients with schizophrenia: a novel once-monthly, long-acting formulation of an atypical antipsychotic. Clin Pharmacokinet 2009, 48:585-600.

17. Invega ${ }^{\circledR}$ Sustenna ${ }^{\circledR}$ (paliperidone palmate) extended-release injectable suspension [prescribing information]. Titusville, NJ: Janssen Pharmaceuticals, Inc; 2012. http://www.janssencns.com/sustenna-prescribing-information. Accessed 30 April 2013.

doi:10.1186/1744-859X-12-22

Cite this article as: Markowitz et al:: Long-acting injectable paliperidone palmitate versus oral paliperidone extended release:

a comparative analysis from two placebo-controlled relapse prevention studies. Annals of General Psychiatry 2013 12:22.

\section{Submit your next manuscript to BioMed Central and take full advantage of:}

- Convenient online submission

- Thorough peer review

- No space constraints or color figure charges

- Immediate publication on acceptance

- Inclusion in PubMed, CAS, Scopus and Google Scholar

- Research which is freely available for redistribution 